\title{
Process of Personalized Prescription of Valproic Acid as the Main Element of the Management of Epilepsy
}

\author{
Elena N. Bochanova, PhD; Natalia A. Shnayder, PhD, ScD*; \\ Diana D. Dmitrenko, PhD, ScD; Ivan P. Artyukhov, PhD, ScD; Sergey D. Gusev, PhD; \\ Elena A. Yurjeva, PhD; Olga S. Shilkina, MD \\ Krasnoyarsk State Medical University named after Prof. V.F. Voino-Yasenetsky \\ Krasnoyarsk, the Russian Federation
}

\begin{abstract}
The purpose of this study was to develop a sequential process of personalized valproic acid (VPA) prescription in patients with epilepsy.

Materials and Methods: We randomly selected 167 patients with epilepsy receiving VPA, based on carriage of CYP2C9*2 and/or CYP2C9*3 and therapeutic drug monitoring. The patients' CYP2C9 status was determined by CYP2C9 genotyping before the beginning of anticonvulsant therapy.

Results: The sequence of personalized valproic acid prescription has been developed.

Conclusion: Using the sequential process of personalized VPA prescription will allow neurologists, psychiatrists and general practitioners to select starting and maintenance dosages of VPA with respect to the individual patient's pharmacogenetic profile and thereby, significantly improve the safety of pharmacotherapy in epilepsy patients.(International Journal of Biomedicine. 2018;8(1):26-32.)
\end{abstract}

Key Words: epilepsy $\bullet$ CYP2C9 $\bullet$ valproic acid $\bullet$ pharmacogenetics $\bullet$ pharmacokinetics

\section{Abbreviations}

ADRs, adverse drug reactions; AEDs, antiepileptic drugs; CYP2C9, cytochrome 2C9; SNPs, single-nucleotide polymorphisms; TDM, therapeutic drug monitoring; VPA, valproic acid.

\section{Introduction}

A personalized approach to the use of VPA, based on carriers of CYP2C9 gene polymorphisms and results of TDM of VPA, can significantly increase treatment safety by reducing the incidence of ADRs from AEDs. ${ }^{(1-3)}$ Desired positive outcomes can only be achieved when a defined sequence of actions is adhered to. However, this sequence is often ambiguous and leads to errors. To avoid this, we utilized block diagrams based on conventional visual programming

*Corresponding author: Prof. Natalia A. Shnayder, PhD, ScD, Head of the Neurological Center of Epileptology, Neurogenetics and Brain Research of the University Clinic of Krasnoyarsk State Medical University named after Prof. V.F. Voino-Yasenetsky, Krasnoyarsk, the Russian Federation.E-mail: nataliashnayder@gmail.com languages to capture the correct sequence of events in VPA

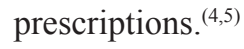

Research into the carriage of polymorphisms of human cytochrome P450 enzymes involved in the metabolism of VPA (CYP2C9, CYP2A6, CYP2B6, CУP1A1, CYP2D6, CYP2E1(1), CYP2E1(2)) showed that the CYP2A6 was the most active in catalyzing VPA 3-hydroxylation whereas CYP2A6 and CYP2B6 were less active. ${ }^{(6)}$

The CYP2C9 gene is highly polymorphic, with more than 50 known alleles. $C Y P 2 C C^{*} 1$ is the wild-type allele and is associated with normal enzyme activity. ${ }^{(7)}$ In carriers of $C Y P 2 C 9 * 2 \quad($ c. $430 \mathrm{C}>\mathrm{T}$; p.Arg144Cys) and $C Y P 2 C 9 * 3$ (c.1075A $>$ C; p.Ile359Leu), the CYP2C9 activity is decreased. ${ }^{(8)}$ The level of toxic metabolites of VPA in human liver microsomes is increased by $28 \%-31 \%$ in samples with one mutated CYP2C9 allele (CYP2C9*2 or CYP2C9*3) and by 
$58 \%-73 \%$ in samples with two mutated CYP2C9 alleles. ${ }^{(9)}$ In carriers of $C Y P 2 C 9 * 2$ and/or $C Y P 2 C 9 * 3$, in comparison with carriers of $C Y P 2 C 9^{*} 1$, there is an achievement of high blood concentrations of VPA, often exceeding the upper level of the therapeutic references, even when taking medium therapeutic doses of this drugs. ${ }^{(10,11)}$ An accumulation of VPA in the blood up to a toxic level can cause the development of serious ADRs due to hyperammonemia, which results from a valproate-induced decrease in L-carnitine in serum. The decrease in L-carnitine is caused by an increase of L-carnitine excretion from urine (in the form of valproilcarnitine) and reduced renal reabsorption of free carnitine and acylcarnitine. L-carnitine deficiency in hepatocytes cases impaired urea synthesis and development of hyperammonemia. ${ }^{(12)}$ Some authors ${ }^{(13,14)}$ showed that the development of ADRs on the background of VPA use was associated with the development of hyperammonemia in patients with no liver diseases. L-carnitine is successfully used to eliminate the ADRs caused by hyperammonemia, including in the treatment of acute valproate overdose. ${ }^{(15)}$ Thus, despite the large amount of information on VPA use in clinical practice, accumulated during more than half a century, pharmacogenetic studies of VPA open up new opportunities for improving the effectiveness and safety of therapy. ${ }^{(16)}$

The purpose of this study was to develop a sequential process of personalized VPA prescription in patients with epilepsy.

\section{Materials and Methods}

We randomly selected 167 patients with epilepsy receiving VPA, based on carriage of $C Y P 2 C 9 * 2$ and/or CYP2C9*3 and TDM status. The patients' CYP2C9 status was determined by CYP2C9 genotyping before the beginning of anticonvulsant therapy. We used a sorption method with a set of reagents for DNA isolation (DNA-Sorb-B) from the clinical material. Genotyping for the CYP2C9*2 (Arg144Cys, C430T) and CYP2C9*3 (Ile359Leu, A1075C) polymorphisms was performed according to previously published research protocols. ${ }^{(2)}$ Block diagrams of processes were constructed using Misrosoft Visio 2013.

\section{Results}

The sequence of personalized VPA prescription was as follows (Figures 1-4). The first step is clinical diagnosis of epilepsy during the first visit to a neurologist, along with ruling out the following contraindications for VPA prescription: an increased sensitivity to any of the components of valproate; acute and chronic hepatitis; severe liver or pancreas dysfunction; hepatic porphyria; established mitochondrial diseases caused by mutations in the mitochondrial enzyme $\gamma$-polymerase (POLG) gene, such as Alpers-Huttenlocher syndrome; urea cycle disorders; patients on mefloquine or Hypericum perforatum preparations, and drugs that increase enzymatic liver activity or disturb intestine function (Fig.1).

After the diagnosis of epilepsy and the decision to start VPA, all patients are genotyped for the $C Y P 2 C{ }^{*} 2$ and CYP2C9*3.
Interpretation of results and VPA dosage are discussed and decided on at the next visit. A starting dose of VPA depends on the homo- or heterozygous carriers of the identified SNPs in CYP2C9. Normal metabolizers (genotype CYP2C $9^{*} 1 / * 1$ ) are prescribed a medium therapeutic dose of VPA. Intermediate metabolizers (heterozygous genotypes CYP $2 C 9 * 1 / * 2$ and CYP $2 C 9 * 1 / * 3$ ) are prescribed a VPA dose $25 \%-30 \%$ lower than average. In poor metabolizers (homozygous genotypes CYP2C9*2/*2 and CYP2C9*3/*3 or compound heterozygotes of $C Y P 2 C 9 * 2 / * 3$ ), the VPA dose is reduced by $50 \%$. The rate of titration of the daily dose of VPA also depends on the identified SNPs of CYP2C9: normal metabolizer - once every 3 days, intermediate metabolizer - once a week, poor metabolizer once every 2 weeks. Patients are assigned a follow-up date with respect to their genotype: normal metabolizer after 6 months, intermediate metabolizer after 3 months, and poor metabolizer after 2 months. All patients are required to conduct VPA TDM prior to their appointment (Fig.1).

Further treatment depends on the results of TDM, clinical effectiveness and ADRs associated with VPA treatment over the last period.

The choice of drug for correction of ADRs of VPA is based on the pathogenetic mechanism of their development. Valproate-induced decrease in the synthesis of L-carnitine in hepatocytes' mitochondria is due to a complex formation between VPA and L-carnitine (valproilcarnitine), which is freely excreted in urine. In addition, reduced renal reabsorption of free carnitine and acylcarnitine leads to a deficiency of L-carnitine, and as a consequence, development of hyperammonemia. ${ }^{(8,9)}$ It is also known that receiving VPA often leads to cholestasis development. ${ }^{(10)}$ The course of hepatoprotective therapy is possible without VPA withdrawal. ${ }^{(11)}$ Prescribing L-carnitine and ursodeoxycholic acid eliminates L-carnitine deficiency and provides a hepatoprotective effect.

For patients with a therapeutic level of VPA TDM (Fig. 2):

- In cases of a positive clinical and electroencephalographic dynamic during treatment, and absence of ADRs, treatment is continued at the same dose.

- In cases of a positive clinical and electroencephalographic dynamic during treatment with development of ADRs, L-carnitine and ursodeoxycholic acid are prescribed for 1 month. VPA dose is reduced by $25 \%$ in patients with ADRs such as aggravation of seizures, thrombocytopenia, hepatopathy, severe alopecia, hand tremor, and significant increase in body weight. In patients with such ADRs as onihodistrophy, non-coarse tremor of the hands and structurally unstable alopecia, and a slight increase in body weight, the therapy continues at the same dose, with the patient's consent.

- Incases of the absence of clinical andelectroencephalographic effect and ADRs development, VPA is discontinued and another AED is prescribed. All patients in this group are prescribed L-carnitine and ursodeoxycholic acid for 1 month.

- In cases where the VPA effect and ADRs, are absent, treatment can be continued with dose escalation by $25 \%$, with the patient's consent. The rate of dose titration is dependent on the individual patient's pharmacogenetic profile. 


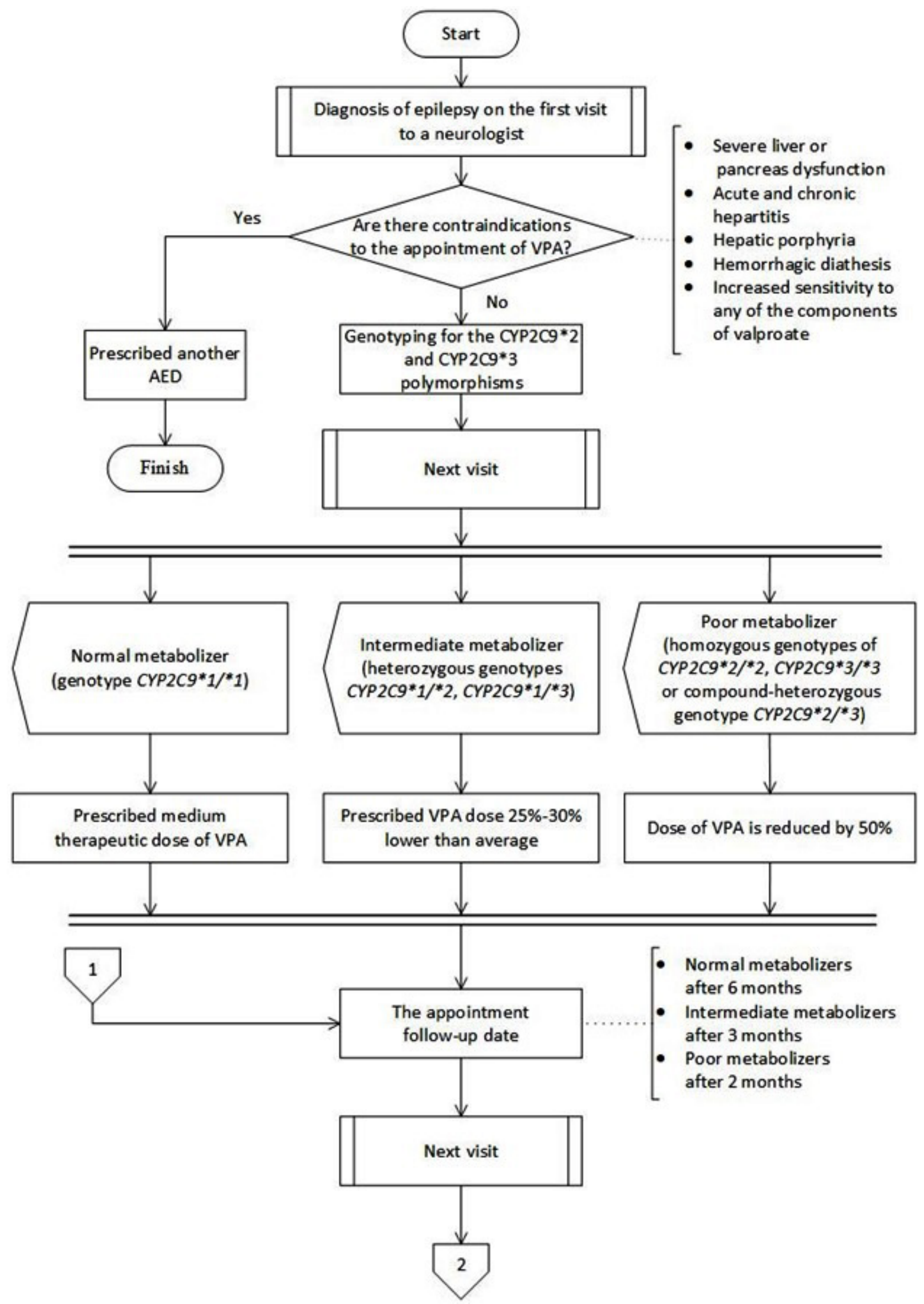

Fig. 1. Sequence of personalized VPA prescription (the start of the process). 


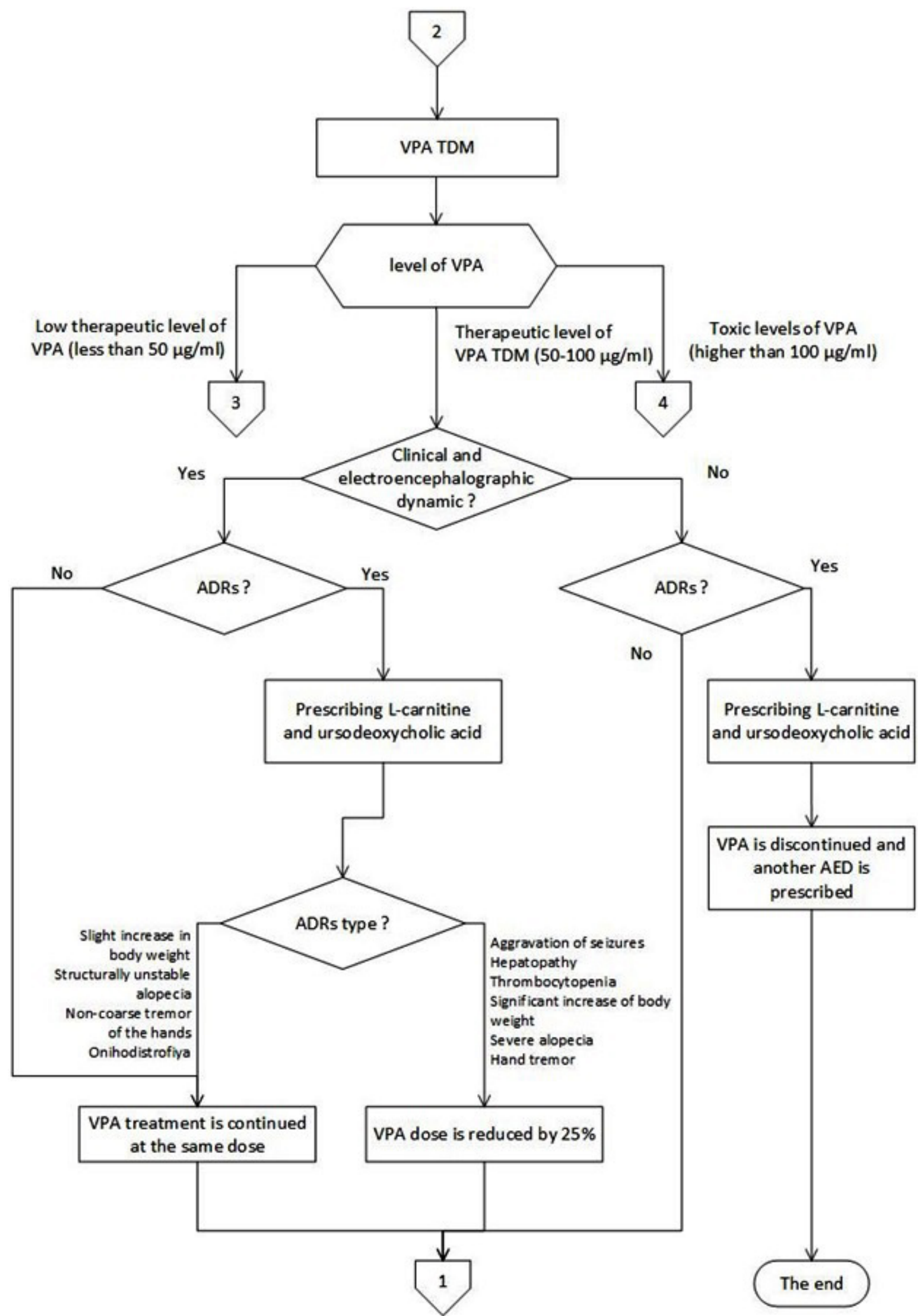

Fig. 2. Process of personalized VPA prescription (continued). 
Patients with a low therapeutic level of VPA $(<50 \mu \mathrm{g} / \mathrm{ml})$ are assessed for compliance with the dosing and scheduling regime of AED administration (Fig.3).

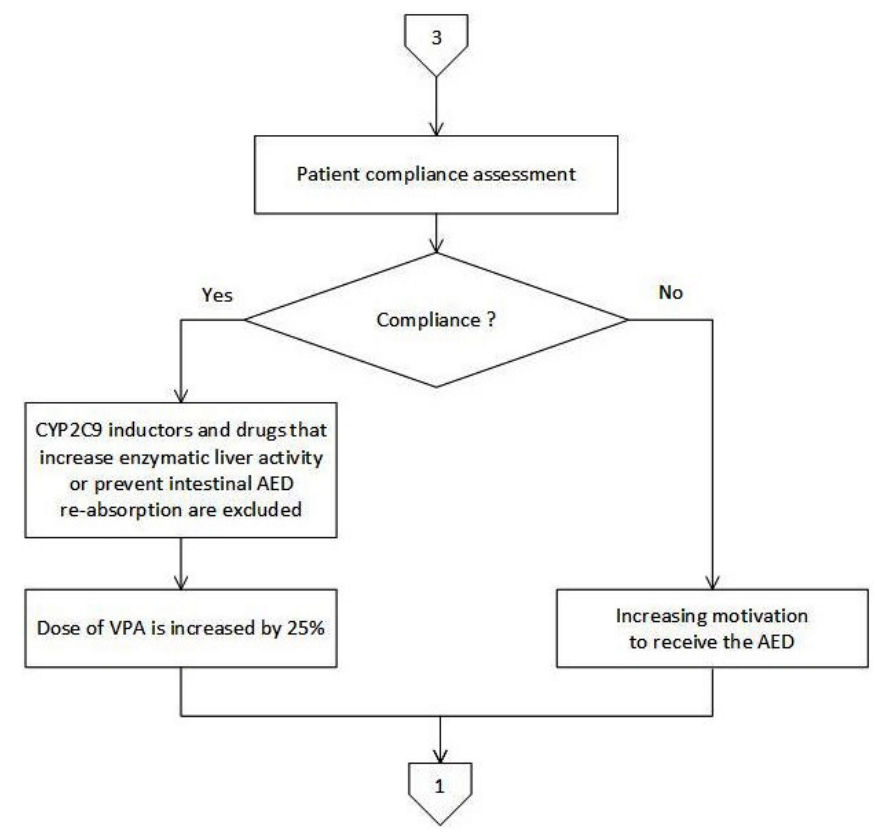

Fig. 3. Process of personalized VPA prescription (continued).

In cases of low patient compliance, motivation to receive the AED is increased and VPA treatment is continued at the same dose.

If a patient follows the recommendations for VPA administration, drugs that increase enzymatic liver activity or prevent intestinal AED re-absorption are excluded. Additional pharmacogenetic examination is advised to exclude profile "normal metabolizer." The dose of VPA is increased by $25 \%$ with the rate of dose titration depending on the individual pharmacogenetic profile of the patient.

Patients with toxic levels of VPA $(>100 \mu \mathrm{g} / \mathrm{ml})$, as a result of TDM, are prescribed L-carnitine and ursodeoxycholic acid for 1 month (Fig.4).

In cases of positive clinical and electroencephalographic dynamic during treatment and the absence of ADRs, the dose of VPA is reduced by $25 \%$.

In cases of a positive clinical and electroencephalographic dynamic during treatment and development of ADRs, the dose of VPA is reduced by $50 \%$ in patients with ADRs such as the aggravation of epileptic seizures, thrombocytopenia, hepatopathy, alopecia and coarse tremor of hands, and a significant increase in body weight. In patients with ADRs in the form of onychodystrophy, non-coarse tremor of the hands and structurally unstable alopecia, and a slight increase in body weight, the treatment dose is reduced by $25 \%$.

In cases where the clinical and electroencephalographic effect of VPA treatment with development of ADRs is absent, VPA is discontinued and another AED is prescribed.

Incases oftheabsenceofclinicalandelectroencephalographic effect of VA without development of ADRs, treatment may be resumed with a dose reduction by $25 \%$, with patient's consent.
In conclusion, using this sequential process of personalized VPA prescription will allow neurologists, psychiatrists and general practitioners to select starting and maintenance dosages of VPA with respect to the individual patient's pharmacogenetic profile and thereby, significantly improve the safety of pharmacotherapy in epilepsy patients.

\section{Competing interests} interests.

\section{References}

1. Dmitrenko D, Shnayder N, Bochanova E, Artyukhov I, Zyryanov S, Veselova O, et al. [Therapeutic drug monitoring in the treatment of epilepsy]. Vrach (The Doctor). 2017;1:8183. [Article in Russian].

2. Shnayder NA, Pilyugina MS, Dmitrenko DV, Bochanova EN, Shapovalova EA, Erikalova SA, et al. Pharmacogenetics of valproic acid as unmodified risk factor of adverse drug reactions. Medical and Health Science Journal. 2011; 7: 2638.

3. Shnaider NA, Sychev DA, Pilyugina MS, Dmitrenko DV, Bochanova EN, Shapovalova EA. Importance of the pharmacokinetics of valproic acid in an individualized approach to the treatment of epileptic women of fertile age. Neuroscience and Behavioral Physiology. 2012;42(9):963968. https://doi.org/10.1007/s11055-012-9663-2

4. Okulova LP. Pedagogical ergonomics: Monograph. Moskow - Ijevsk: Institute of Computer Research; 2011. [In Russian].

5. Parondjanov VD. Learn to write, read and understand algorithms. Algorithms for the thinking. Fundamentals of algorithmization. Moscow: DMK Press; 2012. [In Russian].

6. Kiang TK, Ho PC, Anari MR, Tong V, Abbott FS, Chang TK. Contribution of CYP2C9, CYP2A6, and CYP2B6 to valproic acid metabolism in hepatic microsomes from individuals with the CYP2C9*1/*1 genotype. Toxicol. Sci. 2006;94(2):261-71.

7. Caudle KE, Rettie AE, Whirl-Carrillo M, Smith LH, Mintzer S, Lee MT, et al. Clinical pharmacogenetics implementation consortium guidelines for CYP2C9 and HLA-B genotypes and phenytoin dosing. Clin Pharmacol Ther. 2014;96(5):542-8. doi: 10.1038/clpt.2014.159.

8. Kukes VG, Grachev SV, Sychev DA, Ramenskaya GV. Drugs metabolism. The scientific basis of personalized medicine. M. GEOTAR-Media; 2008. [in Russian].

9. Ho PC, Abbott FS, Zanger UM, Chang TK. Influence of CYP2C9 genotypes on the formation of a hepatotoxic metabolite of valproic acid in human liver microsomes. Pharmacogenomics J. 2003;3(6):335-42.

10. Brusturean-Bota ES, Trifa AP, Coadă CA, Buzoianu $\mathrm{AD}$, Perju-Dumbravă L..Impact of CYP2C9 genetic polymorphisms on valproate dosage, plasma concentrations of valproate and clinical response to valproate. HVM Bioflux. 2013;5(3):94-8.

11. Tan L, Yu JT, Sun YP, Ou JR, Song JH, Yu Y. The influence of cytochrome oxidase CYP2A6, CYP2B6, and CYP2C9 polymorphisms on the plasma concentrations of valproic acid in epileptic patients. Clin Neurol Neurosurg. 2010;112(4):3203. doi: 10.1016/j.clineuro.2010.01.002. 


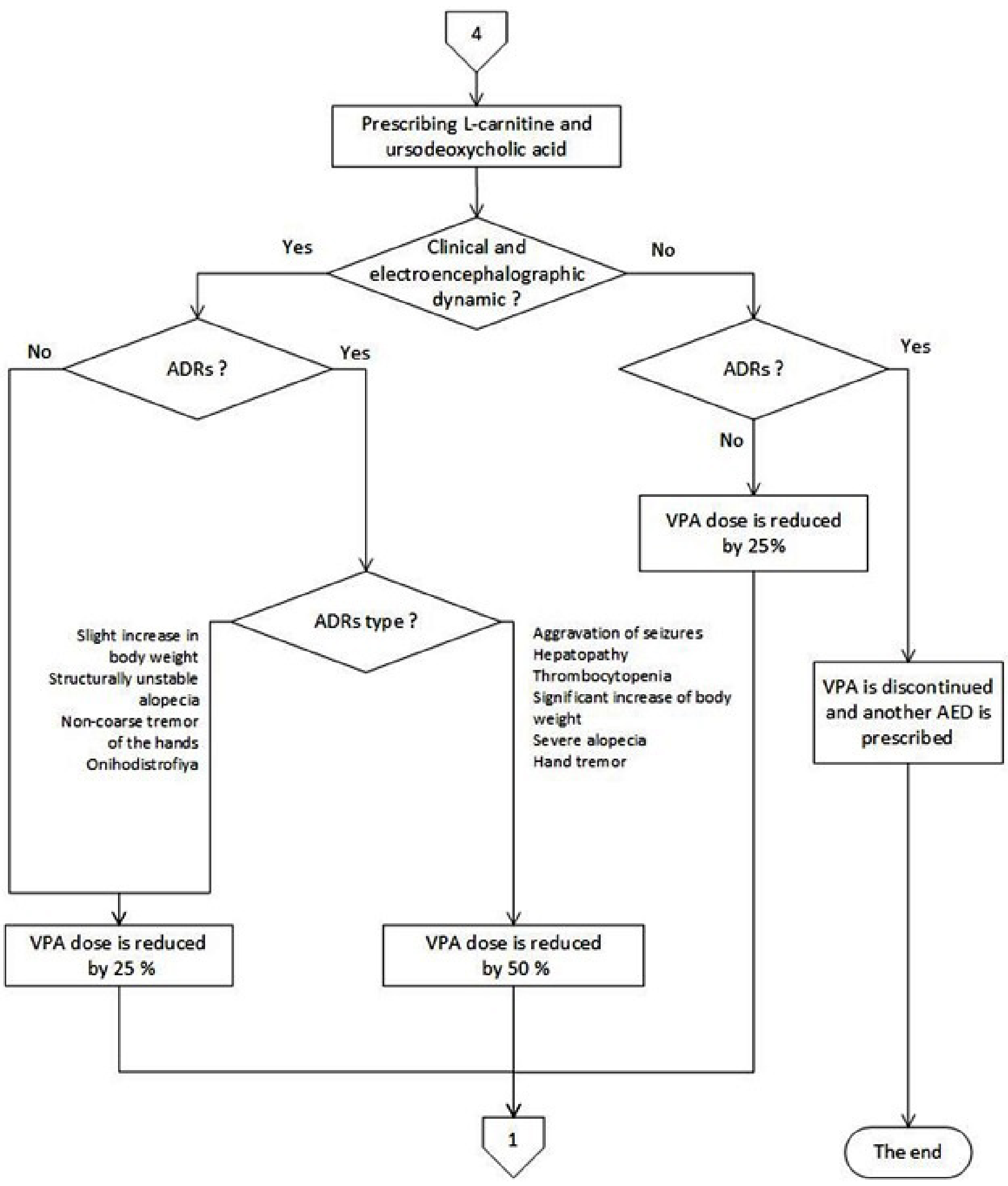

Fig. 4. Process of personalized VPA prescription (continued). 
12. LoVecchio F, Shriki J, Samaddar R. L-carnitine was safely administered in the setting of valproate toxicity. Am J Emerg Med. 2005;23(3):321-2.

13. Blackford MG, Do ST, Enlow TC, Reed MD. Valproic Acid and topiramate induced hyperammonemic encephalopathy in a patient with normal serum carnitine. J Pediatr Pharmacol Ther. 2013;18(2):128-36. doi: 10.5863/1551-6776-18.2.128.

14. Mojumder DK, De Oleo RR. Differential ammonia decay kinetics indicates more than one concurrent etiological mechanism for symptomatic hyperammonemia caused by valproate overdose. Indian J Pharmacol. 2014; 46(3):345-7. doi: 10.4103/0253-7613.132200.

15. Rousseau MC, Montana M, Villano P, Catala A, Blaya $\mathrm{J}$, Valkov M, et al. Valproic acid-induced encephalopathy in very long course treated patients. Brain Inj. 2009;23(12):9814. doi: 10.3109/02699050903302344.

16. Bűdi T, Tóth K, Nagy A, Szever Z, Kiss Á, Temesvári M, et al. Clinical significance of CYP2C9-status guided valproic acid therapy in children. Epilepsia. 2015; 56(6):849-55. doi: 10.1111/epi.13011. 